\title{
ON THE MODELLING CROWD DYNAMICS FROM SCALING TO HYPERBOLIC MACROSCOPIC MODELS
}

\author{
NICOLA BELLOMO \\ Department of Mathematics, Politecnico, Torino, \\ Corso Duca degli Abruzzi 24, 10129 Torino, Italy \\ nicola.bellomo@polito.it \\ CHRISTIAN DOGBÉ \\ Department of Mathematics, University of Caen, \\ CNRS UMR 6139 BP 5186, F-14032 Caen, France \\ dogbe@math.unicaen.fr \\ Received 7 February 2008 \\ Revised 8 April 2008 \\ Communicated by F. Brezzi
}

\begin{abstract}
This paper, that deals with the modelling of crowd dynamics, is the first one of a project finalized to develop a mathematical theory refereing to the modelling of the complex systems constituted by several interacting individuals in bounded and unbounded domains. The first part of the paper is devoted to scaling and related representation problems, then the macroscopic scale is selected and a variety of models are proposed according to different approximations of the pedestrian strategies and interactions. The second part of the paper deals with a qualitative analysis of the models with the aim of analyzing their properties. Finally, a critical analysis is proposed in view of further development of the modelling approach. Additional reasonings are devoted to understanding the conceptual differences between crowd and swarm modelling.
\end{abstract}

Keywords: Crowd dynamics; multiscale; hyperbolic models; nonlinearity; qualitative analysis.

AMS Subject Classification: 91D10, 92D25, 90B20

\section{Introduction}

In the recent years, the interest in complex systems, i.e. systems of several individuals interacting in a nonlinear manner, is greatly increased. These systems are difficult to model or understand at a global level based only on the description of the dynamics of individual elements.

This interest is due to a rising awareness that many systems in nature are of this kind and cannot be successfully modelled by traditional methods developed for inert matter. Moreover, an increasing number of applications in engineering and 
social sciences resemble such systems, given the high number of elements and the complex interactions among them, especially in fields such as transportation and communication networks, social and economic networks.

Complex systems may differ greatly in their nature, but they share one common aspect: the behavior of the system is difficult to understand no matter how simple the behavior of its parts, even though a global pattern or structure certainly occurs. Sometimes this behavior corresponds to the movement of the system in an appropriate abstract configuration space (as, for example, in neural networks). In other cases, the system takes different shapes in a two- or three-dimensional space, and the formation and evolution of characteristic and complex patterns can be observed.

This latter possibility, where the configurations of the system may be interpreted geometrically, is very interesting, because it allows a graphic representation of the evolution of the system, which can be extremely useful to identify some of the relevant characteristics. For instance in crowd dynamics, architects are interested in understanding how crowds move into buildings to find optimality criteria for space design. Transportation engineers face the problem of integration of transportation facilities, with particular emphasis on safety issues for pedestrians. Recent tragic events have increased the interest for modelling pedestrians' movements. In this spirit, it is important to define mathematical models based on specific (and contextdependent) behavioral assumptions, tested by means of proper statistical methods.

This paper deals with the modelling of crowd dynamics in bounded domains, with the aim of developing a general mathematical theory by an approach which includes the ability of the interacting individuals to follow specific strategies also generated by their interaction with the outer environment. The dynamics is remarkably influenced by environmental conditions, for instance by the onset of panic situations. Modifications of these conditions can substantially change the rules of interactions.

It is a fascinating, however difficult topic, in which applied mathematicians and physicists are increasingly interested. However, despite this phenomenon only preliminary results are available in papers unlike those pertaining to similar systems such as vehicular traffic on roads and network of roads. According to the above reasoning, it is worth identifying a few additional complexifying aspects. Among various others:

(i) the dynamics is in two or three space dimensions, while traffic flow is in one space dimension;

(ii) all drivers have approximately the same strategy, which is not consistently modified by outer conditions, while, in crowds, the dynamics of the interactions and the overall strategy is modified according to specific situations, for instance the presence of panic may change them consistently.

Some papers are available mainly concerning modelling issues at the microscopic and macroscopic scales, among others, Henderson ${ }^{13,},{ }^{14}$ Helbing $^{15},{ }^{16}$ Helbing and coworkers ${ }^{17-} ;{ }^{19}$ Hoogendoorn and Bovy ${ }^{21}$ Coscia and Canavesio. ${ }^{7}$ Recent papers 
by Hughes, ${ }^{22,23}$ which deal with macroscopic type modelling, put clearly in evidence that the modelling can be developed only if the thinking ability of interacting individuals is often carefully taken into account. In modelling dynamics of crowds motivation is also related to the control of panic situations or engineering structural safety as documented in the paper by Venuti et al. ${ }^{30}$

The contents of this paper in some ways related to traffic flow modelling documented in the review papers, by Helbing, ${ }^{20}$ Bellomo et al., ${ }^{5}$ Klar et al. ${ }^{24}$ which is far more developed than that of crowds and swarms dynamics. Some recent papers on vehicular traffic flow modelling by kinetic theory with discrete velocities, ${ }^{8,11}$ provide useful modelling of microscopic interactions that can be used also at the macroscopic level as well as to model pedestrian flows.

This paper is the first one of series finalized to develop the various aspects of crowd modelling from scaling to derivation and simulations of models at the various scales. The analysis is mainly focused on modelling crowd dynamics by hydrodynamic equations, while the additional technical difficulties in the modelling of swarms are subsequently put in evidence. The contents are developed through five more sections as follows.

Section 2 deals with the first step of the modelling process, namely the identification of the observation scales to represent the system. As we shall see, it is not naively claimed that the selection of a scale among those that are reported in the section is fully satisfactory as various criticisms can be raised due to the complexity of the system.

Section 3 initiates the modelling approach by assessing the mathematical framework suitable to act as a background structure for the derivation of specific models. The framework consists in the equations of conservation of mass and equilibrium of linear momentum characterized by an acceleration term to be properly modelled.

Section 4 completes the modelling process by offering different types of acceleration terms, that generate different models corresponding to increasing levels of accuracy to capture the essence of crowd dynamics.

Section 5 proposes a qualitative analysis of the various models offered in Sec. 4, focused on the hyperbolicity analysis to characterize the qualitative behavior of the solutions. Numerical simulations, that will appear in Ref. 12, visualize the dynamics of the crowds also referred to some of the calculations reported in this section.

Section 6 deals with critical analysis concerning various aspects of the modelling of crowd dynamics regarded as research perspectives. Specifically, this section deals with the modelling of boundary conditions, with the analysis of the transition from normal to panic conditions, and of the conceptual differences between crowd and swarms modelling.

It is worth stressing that, although this paper deals with the approach by methods of continuum mechanics, the authors are aware that the selection of the macroscopic scale may not even be the most appropriate way to describe the system under consideration. Possibly, different scales and hybrid modelling approaches have to be developed. This paper refers to the project of developing a general theory where 
different mathematical methods are developed for each scale with respect to the other is linked to specific applications.

\section{Scaling and Representation of Crowd Dynamics}

The first step in modelling real systems is the identification of the observation and modelling scales. Subsequently, for each scale one has to identify the parameters and the variables to be used toward modelling. Although, this paper deals with the modelling only by macroscopic hydrodynamic equations, it is worth dealing with the above issue in view of further developments. Classically, the following types of description can be considered:

Microscopic description: All pedestrians, regarded as particles, are individually identified. Position and velocity, regarded as dependent variables of time, of all individuals define the state of the whole system.

Kinetic description: The state of the system is identified by a suitable probability distribution over the microscopic state of the test individual representative of the whole system.

Macroscopic description: The state is described by locally averaged quantities, namely density, mass velocity and energy, regarded as dependent variables of time and space.

In details, let us consider a large system of individuals, regarded as active particles, over a two-dimensional domain $\Omega \in \mathbb{R}^{2}$, which may be either bounded or unbounded. The following parameters can be used toward the identification of dimensionless independent and dependent variables at the various scale:

- $\ell$ is a characteristic length of the system. If $\Omega$ is bounded, $\ell$ is the largest dimension; if $\Omega$ is unbounded, then $\ell$ is the largest dimension of the domain containing the initial localization of the crowd.

- $n_{M}$ is the maximum density of the crowd corresponding to their admissible packing;

- $V_{M}$ is the maximum admissible mean velocity of crowd which may be reached, in average, in free flow conditions, while the maximum admissible velocity for an isolated individual, may be larger that $V_{M}$ is denoted by $(1+\mu) V_{M}, \mu>0$.

The above quantities allow the assessment of the following independent variables:

- $t=t_{\mathrm{r}} / T_{\mathrm{C}}$, where $t_{\mathrm{r}}$ is the real time, is the dimensionless time variable referred to the critical time $T_{\mathrm{C}}=V_{M} / \ell$.

- $x=x_{\mathrm{r}} / \ell$, and $y=y_{\mathrm{r}} / \ell$ which are the dimensionless space variables obtained referring to the real space variables $x_{\mathrm{r}}$ and $y_{\mathrm{r}}$ to $\ell$.

After the above preliminary definitions, it is possible to assess the variables to represent the system at the various scales. 
The microscopic representation is defined by the following variables:

$\mathbf{x}_{i}=\{x, y\}_{i}$, which identifies, for $i=1, \ldots, N$, the position in $\Omega$ of each $i$ th individual of a crowd of $N$ individuals;

$\mathbf{V}_{i}=\left\{V_{x}, V_{y}\right\}_{i}$, which identifies the dimensionless (being referred to $V_{M}$, velocity of each $i$ th individual of the crowd.

Mathematical models are generally stated as a system of $N$ ordinary differential equations where $\mathbf{v}_{i}$ and $\mathbf{x}_{i}$ are the dependent variables, that are normalized with respect to $V_{M}$ and $\ell$, respectively.

The kinetic (statistical) representation of a system constituted by a large number of interacting individuals is defined by the statistical distribution of their position and velocity:

$$
f=f(t, \mathbf{x}, \mathbf{V})=f\left(t, x, y, V_{x}, V_{y}\right),
$$

where, if $f$ is locally integrable, $f(t, \mathbf{x}, \mathbf{V}) d \mathbf{x} d \mathbf{V}$ denotes the number of individuals, which, at the time $t$, are in the elementary domain of the microscopic states $[x, x+$ $d x] \times[y, y+d y] \times\left[V_{x}, V_{x}+d V_{x}\right] \times\left[V_{y}, V_{y}+d V_{y}\right]$.

The distribution function $f$ can be normalized with respect to $n_{M}$, while also in this case the microscopic variables are normalized with respect to $V_{M}$ and $\ell$, respectively. Therefore, all derived variables can be given in a dimensionless form. Macroscopic observable quantities can be obtained, under suitable integrability assumptions, by moments of the distribution. In particular, the dimensionless local density is given by

$$
\rho(t, \mathbf{x})=\int_{0}^{1+\mu} \int_{0}^{1+\mu} f(t, \mathbf{x}, \mathbf{V}) d \mathbf{V} .
$$

The total number of individuals in $\Omega$, at time $t$, is given by

$$
N(t)=\int_{\Omega} \rho(t, \mathbf{x}) d \mathbf{x}
$$

Analogously, the mean velocity can be computed as follows:

$$
\vec{v}(t, \mathbf{x})=E[\mathbf{V}](t, \mathbf{x})=\frac{1}{\rho(t, x)} \int_{0}^{1+\mu} \int_{0}^{1+\mu} \mathbf{V} f(t, \mathbf{x}, \mathbf{V}) d \mathbf{V},
$$

and similarly the speed variance

$$
\sigma(t, \mathbf{x})=\frac{1}{\rho(t, \mathbf{x})} \int_{0}^{1+\mu} \int_{0}^{1+\mu}[\mathbf{V}-E[\mathbf{V}](t, \mathbf{x})]^{2} f(t, \mathbf{x}, \mathbf{V}) d \mathbf{V}
$$

where the speed variance provides a measure of the stochastic behavior of the system with respect to the deterministic macroscopic description.

Mathematical models obtained, in the framework of the kinetic theory, by evolution equations for the above defined distribution function obtained by number density balance in the elementary volume of the space of the microscopic states. Inflows and outflows into and from such a volume are determined by interactions between the test individual and the field ones. 
The mathematical kinetic theory for active particles, see Ref. 2, suggests adding to the modelling of the microscopic state an additional activity variable suitable to describe the strategy of each individual regarded as an active particle.

The macroscopic representation of a system constituted by a large number of interacting individuals concerns groups of pedestrians rather than the individual units. Macroscopic representation may be selected for high density, large scale systems in which the local behavior of groups is sufficient.

In details, the macroscopic representation is defined by the following variables: $\rho=\rho(t, x, y)$ which is the dimensionless density referred the local number density $n=n(t, x, y)$ to the maximum admissible density $n_{M}$;

$\vec{v}=\vec{v}(t, x, y)$ that is the dimensionless mean velocity, referred to $V_{M}$, that, in two space dimensions, expressed by the unit vectors denoted by $(\vec{i}, \vec{j})$, writes:

$$
\vec{v}(t, x, y)=v_{x}(t, x, y) \vec{i}+v_{y}(t, x, y) \vec{j}
$$

The relationship between the flow rate, the mean velocity and the pedestrian density is given, in dimensionless form, as follows: $\vec{q}=\rho \vec{v}$.

\section{Macroscopic Frameworks Toward Modelling}

This section deals with the derivation of the mathematical framework suitable to act as a general paradigm for the derivation of specific models. Classically, equation of conservation of mass and equilibrium of linear momentum can be used. The main conceptual difficulty consists in modelling the closure of momentum equation by suitable phenomenological models of the acceleration applied to individuals in the elementary volume $d \mathbf{x}=d x d y$.

Pedestrian movement shows characteristics different from those of cars or other vehicles. Pedestrians have more flexibility to move in two dimensions, as well as more flexibility to stop and go within the full range of admissible velocities, that is not the case of vehicles. This is due to the wide domain of visibility area controlled by them. However, the hydrodynamic approach refers to locally averaged quantities, therefore local fluctuations in the velocity are not modelled explicitly.

Bearing all the above in mind, let us consider the modelling approach offered by continuum mechanics that approximates the system under consideration as a continuum flow. Therefore, if the distances between the crowds are assumed to be negligible. According to Sec. 2, the state of the system, in two space dimensions, is described by density of the crowd and the average speed: $\rho=\rho(t, x, y)$ and $\vec{v}=\vec{v}(t, x, y)$, while the local flow is given by:

$$
\vec{q}=\vec{q}(t, x, y)=\rho(t, v, y) \vec{v}(t, v, y)
$$

where, according to the continuum approach, the above quantities are supposed to be differentiable with respect to the dependent variables. 


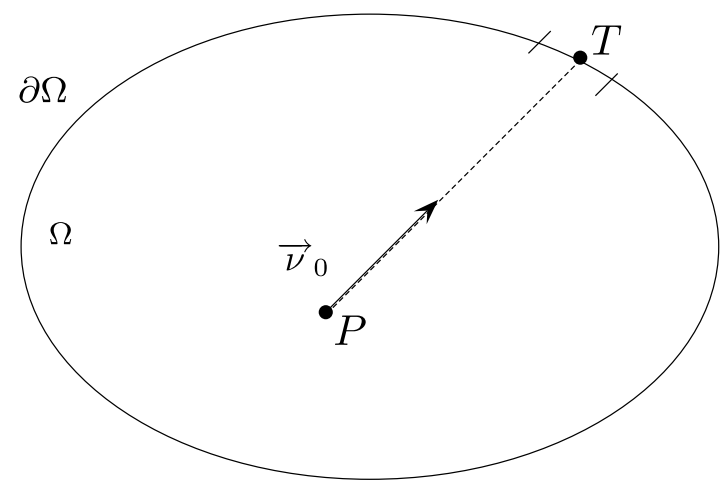

Fig. 1. Geometry of the domain occupied by the crowd.

Referring to Fig. 1, let us consider, the crowd in a bounded domain $\Omega \subset \mathbb{R}^{2}$, where $\partial \Omega$ is its boundary. The overall description of the system is delivered by the equation of conservation of mass and equilibrium of linear momentum defined by the following system of partial differential equations:

$$
\left\{\begin{array}{l}
\partial_{t} \rho+\nabla_{\mathbf{x}} \cdot(\rho \vec{v})=0 \\
\partial_{t} \vec{v}+\vec{v}\left(\nabla_{\mathbf{x}} \cdot \vec{v}\right)=\vec{F}[\rho, \vec{v}],
\end{array}\right.
$$

where $\vec{F}$ models the average acceleration that acts over the elementary block of individuals in volume $d x d y$. Here $\partial_{t}$ stands for the partial time derivative. The notation $(\cdot)_{\phi}=\frac{\partial(\cdot)}{\partial \phi}$ will be used here after. The first equation being pedestrian conservation and the second speed dynamics; brackets are used to indicate that in Eq. (3.1), $\vec{F}=\left\{F_{x}, F_{y}\right\}$ can be, in specific models, a functional of its arguments, for instance it can be a function not only from $\rho$ and $\vec{v}$ but also of their space derivative.

Remark 3.1. The above system acts as the general framework for the derivation of specific models which can be classified as first-order models if only the mass conservation equation is used and is properly closed by a phenomenological model linking the velocity to the local density conditions (including density gradients), while second-order models refer to the whole system and are obtained by closing the linear momentum equation by a phenomenological model of the term $\vec{F}$.

An example of a first-order model has been recently proposed in a paper. ${ }^{7}$ Additional studies in one space dimension for crowds in on platforms have been given in the paper by Venuti et al. ${ }^{30}$ The remaining part of this paper is devoted to the derivation and analysis of second-order models. Some preliminary remarks are given below in view of the detailed modelling proposed in the next section.

Remark 3.2. $\vec{F}$ is not the real physical force applied by an external field; it characterizes the internal driving force or motivation of the pedestrian. That is why, we 
talk about acceleration, considering that using the term "force" is not appropriate for a system in which the "mass" cannot be properly defined.

Remark 3.3. Acceleration in models of crowds is a basic element that must be represented for three reasons. First, acceleration has a direct effect on movement: people in a crowd can be pushed around. Second, acceleration is a perceptible input to the cognitive system and a major source of information in an informationstarved situation. Third, acceleration carries the consequences of dangerous crowd scenarios: injuries.

Remark 3.4. A preliminary observation, still waiting for the derivation of models, is that pedestrians have a target to reach, for instance a point $T$ of the boundary corresponding to the exit. Therefore, given a point $P=\{x, y\}$ inside $\Omega$ is useful, for the calculations developed in the next section, defining the unit vector from $P$ to the target $T$ as shown in Fig. 1.

The calculation of the unit vector $\vec{\nu}_{0}$, according to the geometry of the system, is simply as follows:

$$
\vec{\nu}_{0}(x, y)=\vec{\nu}_{x 0}(x, y)+\vec{\nu}_{y 0}(x, y)
$$

where

$$
\vec{\nu}_{x 0}(x, y)=\frac{x-x_{T}}{\sqrt{\left(x-x_{T}\right)^{2}+\left(y-y_{T}\right)^{2}}} \vec{i},
$$

and

$$
\vec{\nu}_{y 0}(x, y)=\frac{y-y_{T}}{\sqrt{\left(x-x_{T}\right)^{2}+\left(y-y_{T}\right)^{2}}} \vec{j},
$$

where the direction of the vector is simply identified by the coordinates of the points $P$ and $T$.

Of course, pedestrians may have a target inside the domain $\Omega$, simple technical modifications are needed to consider this case. The statement of mathematical problems needs suitable boundary conditions unless the modelling refers to crowds in unbounded domains. However, it is still reasonable for the crowd to have a target.

\section{Second-Order Models}

This section deals with the derivation of second-order models, namely models with accelerations. All models consist of two equations given in a $2 \mathrm{D}$ system of partial differential equations (3.1) with a phenomenological relation that describes the average $2 \mathrm{D}$ acceleration by which the crowd modifies its own speed: $\vec{F}=\left\{F_{x}, F_{y}\right\}$, where the components of $\vec{F}$ may depend on the local density, density gradients, velocity and position of the crowd: $\vec{F}=\vec{F}\left[\rho, \vec{v}, \nu_{0}\right]$, where square brackets denote functional dependence.

Different classes of models can be identified according to different ways of modelling the aforementioned acceleration. Specifically, two classes of models here are 
considered simply identified by the way pedestrian select their direction of motion. In details:

Class I: The first class refers to systems where the pedestrians move along straight lines toward the target objective.

Class II: The second class of models refers to walkers that still move toward the target objective, but are also attracted by paths with small density gradients.

Class III: The third class of models contains a "pressure" term which enables the momentum equation to predict the expect response of crowd behavior as time and space changes.

The following two subsections deal with modelling issues related to the above classification, while the third subsection reports about some technical developments involving a modification of the conservation equation. For instance, according to the some natural development of the Aw and Rascle model ${ }^{1}$ which use, as we shall see, a different dependent variable.

Remark 4.1. The above classification simply takes into account the direction followed by pedestrians, while different expression of the acceleration along such a direction can be proposed, as we shall see, according to their specific phenomenological behaviors.

Remark 4.2. The modelling is proposed in normal flow conditions. The dynamics in panic conditions is analyzed in the last section. The presentation of the various models follows the same guidelines: the fundamental assumptions that generate each class of models is stated, subsequently the mathematical framework is derived, and finally same specific models are derived based on suitable phenomenological assumptions concerning the acceleration term.

\subsection{Class $I$}

Let us consider, referring to Fig. 1, a class of models derived according to the following hypothesis:

Assumption 1. Pedestrians seek to minimize their (accurately) estimated travel time but temper their velocity according to local density. Specifically, in each point $P=\left\{x_{P}, y_{P}\right\}$ of the domain, individuals move toward a given objective along the direction $\vec{\nu}_{0}(x, y)$. Moreover, their acceleration consists of two contributions: the first one corresponding to a trend and to equilibrium velocity depending on the local density, directed toward $\vec{\nu}_{0}(x, y)$, and the second term to the action of the density gradients toward $\vec{\nu}_{0}$. In particular, negative values increase the acceleration, while positive values decrease it. 
The formal structure of the system corresponding to mass conservation and linear momentum equilibrium is as follows:

$$
\left\{\begin{array}{l}
\partial_{t} \rho+\partial_{x}\left(\rho v_{x}\right)+\partial_{y}\left(\rho v_{y}\right)=0 \\
\partial_{t} v_{x}+v_{x} \partial_{x} v_{x}+v_{y} \partial_{y} v_{x}=F_{1 x}(\mathbf{x}, \rho, \vec{v})+F_{2 x}\left(\mathbf{x}, \rho, \nabla_{\nu_{0}} \rho\right), \\
\partial_{t} v_{y}+v_{x} \partial_{x} v_{y}+v_{y} \partial_{y} v_{y}=F_{1 y}(\mathbf{x}, \rho, \vec{v})+F_{2 y}\left(\mathbf{x}, \rho, \nabla_{\nu_{0}} \rho\right),
\end{array}\right.
$$

where the first term corresponds to the adaptation to the velocity $\vec{v}_{e}$, which depends on the local density, while the second term corresponds to the influence of local density gradients.

The above acceleration terms can be specialized as follows:

$$
\vec{F}_{1}=\alpha\left(v_{e}(\rho) \vec{\nu}_{0}-\vec{v}\right),
$$

where $\alpha$ is a constant representing the inverse of the relaxation time of $\vec{v}$ toward the generalized equilibrium velocity $v(\rho) \vec{\nu}_{0}$ and

$$
\vec{F}_{2}=-\frac{K^{2}(\rho)}{\rho} \nabla_{\nu_{0}} \rho
$$

Inserting the above formal expression into (4.1) generates the following vector system:

$$
\left\{\begin{array}{l}
\partial_{t} \rho+\nabla_{\mathbf{x}} \cdot(\rho \vec{v})=0, \\
\partial_{t} \vec{v}+\left(\vec{v} \cdot \nabla_{\mathbf{x}}\right) \vec{v}=\alpha\left(v_{e}(\rho) \vec{\nu}_{0}-\vec{v}\right)-\frac{K^{2}(\rho)}{\rho} \nabla_{\nu_{0}} \rho .
\end{array}\right.
$$

Different specific models can be derived in agreement with (4.4) according to different specific models (4.2) and (4.3). In particular, various models have been proposed in the mathematical literature for vehicular traffic flow to describe the trend defined in (4.2). The simplest model is based on the assumption of a linear decay: $v_{e}(\rho)=1-\rho$. However, this extremely simple model does not take properly into account as the quality of the environment, as the decay with respect to density depends on its quality. It may be less steepest when the quality of the environment is good. The model proposed in Ref. 5 to stimulate equilibrium vehicular traffic flow is as follows:

$$
v_{e}(\rho)=\exp \left(-c_{1} \frac{\rho}{1-\rho}\right)
$$

while the so-called Kladek model reported in Ref. 30 refers specifically to pedestrian flow:

$$
v_{e}(\rho)=1-\exp \left(-c_{2} \frac{1-\rho}{\rho}\right) .
$$

Various models can be derived by using different expressions of $K^{2}(\rho)$. For instance:

(1) with $K^{2}(\rho)=0$

$$
\partial_{t} \vec{v}+\left(\vec{v} \cdot \nabla_{\nu_{0}}\right) \vec{v}=\alpha\left(v_{e}(\rho) \vec{\nu}_{0}-\vec{v}\right)
$$


(2) for $K^{2}(\rho)=\beta_{1}^{2}$, we deduce

$$
\partial_{t} \vec{v}+\left(\vec{v} \cdot \nabla_{\nu_{0}}\right) \vec{v}=\alpha\left(v_{e}(\rho) \vec{\nu}_{0}-\vec{v}\right)-\frac{\beta_{1}^{2}}{\rho} \nabla_{\nu_{0}} \rho
$$

(3) as in analogy to the traffic vehicular, ${ }^{25}$ we have $K^{2}(\rho)=\beta_{2}^{2} \rho^{2}\left(\left\|\vec{v}_{e}\right\|^{2}\right)$ which generates

$$
\partial_{t} \vec{v}+\left(\vec{v} \cdot \nabla_{\nu_{0}}\right) \vec{v}=\alpha\left(v_{e}(\rho) \vec{\nu}_{0}-\vec{v}\right)-\beta_{2}^{2} \rho\left(\left\|\vec{v}_{e}\right\|^{2}\right) \nabla_{\nu_{0}} \rho
$$

as in models by Zhang ${ }^{31}, ;^{32}$

(4) the choice of $K^{2}(\rho)=\beta_{3}^{2} \alpha\left\|\vec{v}_{e}\right\|$ yields

$$
\partial_{t} \vec{v}+\left(\vec{v} \cdot \nabla_{\nu_{0}}\right) \vec{v}=\alpha\left(v_{e}(\rho) \vec{\nu}_{0}-\vec{v}\right)-\beta_{3}^{2} \rho\left\|\vec{v}_{e}\right\| \nabla_{\nu_{0}} \rho
$$

as in the model by Payne; ${ }^{28}$

(5) the use of the relation $K^{2}(\rho)=-\rho\left\|\vec{v}_{e}\right\| e^{\alpha\left\|\vec{v}_{e}-\vec{v}\right\|}$ generates:

$$
\partial_{t} \vec{v}+\left(\vec{v} \cdot \nabla_{\nu_{0}}\right) \vec{v}=\alpha\left(v_{e}(\rho) \vec{\nu}_{0}-\vec{v}\right)-\left\|\vec{v}_{e}\right\| e^{\alpha\left\|\vec{v}_{e}-\vec{v}\right\|} \nabla_{\nu_{0}} \rho
$$

as in the model by Del Castillo and Benitez. ${ }^{10}$

The above class of models can be further refined by taking the gradients along the local mean velocity. It is however a technical difference that does not introduce significant additional improvements.

\subsection{Class II}

The class of models proposed in the preceding subsection is such that pedestrians direct their motion, from any point $P$ of the domain $\Omega$, to the target $T$. On the other hand, the direction of motion generally takes into account the fact that pedestrians attempt to avoid zones with higher density, while the selection of optimal paths occurs only in their visibility zone. The reasoning has some analogy with that used in the modelling of pedestrian flow by first-order models. ${ }^{7}$

Conservation equations (4.1) or (4.4) are still used however the direction identified by the unit vector $\nu_{0}$ must be substituted by a new one that takes into account the above phenomena. Specifically, referring to Figs. 1 and 2, the following assumption is proposed:

Assumption 2. Pedestrians seek to join the objective by undervalying their time if possible and at the same time while seeking to avoid the zones with high density. Pedestrians do not have a global vision of the situation, but their perception of the density is limited visual field.

The formalization of the above assumption into mathematical terms needs the identification of the path-direction $\nu$ and of the visibility zone, bearing in mind that the above two quantities are technically related.

Bearing all above in mind let us define by

$$
\gamma=\arctan \left(\frac{\nu_{0 y}}{\nu_{0 x}}\right)
$$




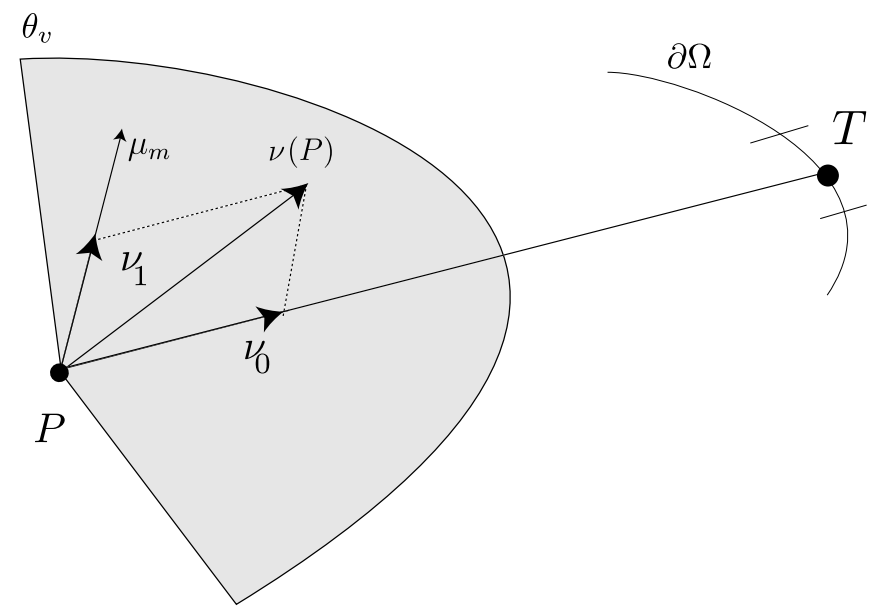

Fig. 2. Target and visibility zone.

the angle which characterizes the direction of the vector $\vec{\nu}_{0}$, and by $\theta_{v}$ the maximum angle of visibility of the pedestrian (in average), one can define a "visual range" interval:

$$
R_{v}=\left[\gamma-\theta_{v}, \gamma+\theta_{v}\right]
$$

to the inside of which the direction toward which heading is chosen. Therefore, let us define the following quantity

$$
I=\left\{\vec{\xi} \cos \alpha \vec{i}+\sin \alpha \vec{j} \mid \alpha \in R_{v}\right\}
$$

and by $\vec{\mu}_{m}=\vec{\mu}_{m}(P)$ the minimal direction of the directional derivative

$$
\min _{\vec{\xi} \in I}\left[\nabla_{x} \rho(P) \cdot \vec{\xi}\right]
$$

and $\vec{\mu}_{M}=\vec{\mu}_{M}(P)$ the direction of maximum of the directional derivative

$$
\max _{\vec{\xi} \in I}\left[\nabla_{\mathbf{x}} \rho(P) \cdot \vec{\xi}\right]
$$

where $I$ is the domain of the path directions on $R_{v}$.

The direction of the motion of pedestrians can be defined, given a density field, as follows:

$$
\vec{\nu}(P)=\vec{\nu}_{0}(P)+\vec{\nu}_{1}(P),
$$

where $\nu_{0}$ has been defined in Sec. 3 and the corrective term is given by

$$
\vec{\nu}_{1}(P)=\eta \vec{\mu}_{m}(P)
$$

where $\eta$ is a positive small parameter introduced to model a corrective term related to the attraction toward small density gradients.

Remark 4.3. The above modelling refers to normal flow conditions, while the situation of panic condition is analyzed in the last section. 
Mathematical models are then related to the following structure:

$$
\left\{\begin{array}{l}
\partial_{t} \rho+\nabla_{\mathbf{x}} \cdot(\rho \vec{v})=0 \\
\partial_{t} \vec{v}+\left(\vec{v} \cdot \nabla_{\mathbf{x}}\right) \vec{v}=\vec{F}[\rho, \vec{v}]=\alpha\left(v_{e}(\rho) \vec{\nu}-\vec{v}\right)-\frac{K^{2}(\rho)}{\rho} \nabla_{\vec{\nu}} \rho,
\end{array}\right.
$$

that uses, with respect to (4.7), the direction $\nu$ instead of $\nu_{0}$.

Specific models are obtained, as in Sec. 4.1, by adopting suitable expressions of the terms $v_{e}$ and $K^{2}(\rho)$. Although technically more complex, the above modelling method has the advantage of leading, as we shall see in the sequel, to an immediate modelling approach of panic conditions.

It is worth stressing that further developments can be obtained by taking into account additional phenomena in the modelling of the acceleration term $\vec{F}[\rho, \vec{v}]$ for instance, a linear velocity diffusion term corresponding to a viscous dissipation in fluid dynamical framework can be added. Consequently, the acceleration of pedestrians will be given by three contributions: the first corresponds to a trend to equilibrium, the second to the action of the density gradient and the third to a dissipative velocity diffusion

$$
\vec{F}[\rho, \vec{v}]=\vec{F}_{1}[\rho, \vec{v}]+\vec{F}_{2}[\rho, \vec{v}]+\vec{F}_{3}[\rho, \vec{v}]=c_{1}\left(\vec{v}_{e}-\vec{v}\right)-\frac{1}{\rho} \nabla_{x} p+\frac{\epsilon}{\rho} \Delta \vec{v},
$$

where $\epsilon$ is a parameter positive which represents in the case of the fluids, the viscosity.

Technical calculations to obtain specific models can be, subsequently, obtained as we have seen above.

\subsection{Class III}

The various models proposed in the preceding section have been obtained by using conservation of mass and linear momentum. The analysis of traffic flow modelling has shown that it can be useful a modelling approach based on the conservation of different quantities such as the total pressure. Our model of Class III is based on a 1D traffic flow model proposed by Aw and Rascle. ${ }^{1}$

The first equation is the two-dimensional conservation law equation given by

$$
\partial_{t} \rho+\partial_{x}\left(\rho v_{x}\right)+\partial_{y}\left(\rho v_{y}\right)=0 .
$$

The second equation is obtained by applying the convective derivative on the pressure terms for the $2 \mathrm{D}$ case and it is given by

$$
\begin{gathered}
\partial_{t}\left(v_{x}+P_{1}\left(\rho, v_{x}\right) \vec{\nu}_{0}\right)+v_{x} \partial_{x}\left(v_{x}+P_{1}\left(\rho, v_{x}\right) \nu_{x_{0}}\right) \\
+v_{y} \partial_{y}\left(v_{x}+P_{1}\left(\rho, v_{x}\right) \nu_{x_{0}}\right)=\rho \mathscr{A}_{1}[\rho, \vec{v}] \\
\partial_{t}\left(v_{y}+P_{2}\left(\rho, v_{y}\right) \vec{\nu}_{y_{0}}\right)+v_{x} \partial_{x}\left(v_{y}+P_{2}\left(\rho, v_{y}\right) \nu_{y_{0}}\right) \\
+v_{y} \partial_{y}\left(v_{y}+P_{2}\left(\rho, v_{y}\right) \nu_{y_{0}}\right)=\rho \mathscr{A}_{2}[\rho, \vec{v}],
\end{gathered}
$$


where $P(\rho, \vec{v})$ is a proposed function given by

$$
P(\rho, \vec{v})=\frac{\rho^{\gamma+1}}{\beta-\rho^{\gamma+1}} \vec{v}
$$

and valid for $\gamma>0$ is a dimensionless constant and $\beta>\rho_{m}^{\gamma+1}$ with $\rho_{m}$ means the maximum density and

$$
\mathscr{A}_{1}[\rho, \vec{v}]=\alpha\left(v_{e}(\rho) \nu_{x_{0}}-v_{x}\right) ; \quad \mathscr{A}_{2}[\rho, \vec{v}]=\alpha\left(v_{e}(\rho) \nu_{y_{0}}-v_{y}\right) .
$$

In a more compact form, the system of Class III can be written as follows:

$$
\left\{\begin{array}{l}
\partial_{t} \rho+\nabla_{x} \cdot(\rho \vec{v})=0, \\
\partial_{t}\left(\vec{v}+P(\rho, \vec{v}) \vec{\nu}_{0}\right)+\left(\vec{v} \cdot \nabla_{x}\right)\left(\vec{v}+P(\rho, \vec{v}) \vec{\nu}_{0}\right)=\overrightarrow{\mathscr{A}}[\rho, \vec{v}],
\end{array}\right.
$$

while, in dimensionless variables, Eq. (4.15) is as follows:

$$
\left\{\begin{array}{c}
\partial_{t} \rho+\rho\left(\partial_{x} v_{x}+\partial_{y} v_{y}\right)+v_{x} \partial_{x} \rho+v_{y} \partial_{y} \rho=0 \\
\partial_{t}\left(v_{x}+P_{1}\left(\rho, v_{x}\right) \nu_{x_{0}}\right)+v_{x} \partial_{x}\left(v_{x}+P_{1}\left(\rho, v_{x}\right) \nu_{x_{0}}\right) \\
\quad+v_{y} \partial_{y}\left(v_{x}+P_{1}\left(\rho, v_{x}\right) \nu_{x_{0}}\right)=\mathscr{A}_{1}[\rho, \vec{v}] \\
\partial_{t}\left(v_{y}+P_{2}\left(\rho, v_{y}\right) \nu_{y_{0}}\right)+v_{x} \partial_{x}\left(v_{y}+P_{2}\left(\rho, v_{y}\right) \nu_{y_{0}}\right) \\
\quad+v_{y} \partial_{y}\left(v_{y}+P_{2}\left(\rho, v_{y}\right) \nu_{y_{0}}\right)=\mathscr{A}_{2}[\rho, \vec{v}]
\end{array}\right.
$$

supplemented by initial conditions $\rho(0, x, y) \geq 0, v_{x}(0, x) \leq\left|v_{1 x}\right|$ and $v_{y}(0, y) \leq$ $\left|v_{2 y}\right|$. Here $v_{1 x}$ and $v_{2 y}$ are the free flow speed.

An additional alternative is obtained by using for the first equation the twodimensional conservation of continuity that conserve mass (pedestrians) given by

$$
\partial_{t} \rho+\partial_{x}\left(\rho v_{x}\right)+\partial_{y}\left(\rho v_{y}\right)=0 .
$$

The flux flow rate in both directions is represented by $\rho v_{x}$ and $\rho v_{y}$. The second equation is similar to the momentum equations in $2 \mathrm{D}$ for compressible flow with some manipulations to mimic crowd dynamics and it is given by

$$
\begin{gathered}
\partial_{t} v_{x}+v_{x} \partial_{x} v_{x}+v_{y} \partial_{y} v_{x}+\rho v_{e}^{\prime}(\rho) \nu_{x_{0}}\left[\partial_{x} v_{x}+\partial_{y} v_{y}\right]=\mathscr{A}_{1}[\rho, \vec{v}], \\
\partial_{t} v_{y}+v_{x} \partial_{x} v_{y}+v_{y} \partial_{y} v_{y}+\rho v_{e}^{\prime}(\rho) \nu_{y_{0}}\left[\partial_{x} v_{y}+\partial_{y} v_{y}\right]=\mathscr{A}_{2}[\rho, \vec{v}],
\end{gathered}
$$

where $\rho v_{e}^{\prime}(\rho)$ and $\rho v_{e}^{\prime}(\rho)$ are the traffic sound speed at which small traffic disturbances are propagated relative to the moving crowd stream. Here, the prime " " is a short-hand notation for total derivative $d / d$. This model will be classified as a crowd flow nonlinear, time varying, hyperbolic system of two partial differential equations.

The above modelling approach can be further developed by following the remarks below.

Remark 4.4. The influence of local gradients can be included analogously to what we have seen for the II-type models. Therefore, the treatment of panic conditions is again as stated in Remark 4.3. 
Remark 4.5. A recent paper by Degond and Delitala ${ }^{9}$ has shown that an appropriate modelling of the invariant, corresponding to momentum and pseudo-pressure, leads to a dynamics with a trend to an equilibrium velocity, versus density, that corresponds to the velocity diagram that is experimentally observed. This avoids introducing artificially such a trend. This approach needs to be properly adapted to modelling also crowd dynamics without using the artificial terms $\mathscr{A}_{1,2}$.

\section{Hyperbolicity Analysis of Crowd Dynamic Models}

Our exposition is completed, in this section, by a qualitative analysis of the partial differential equations (PDEs) used in the modelling approach. Specifically, one should expect models characterized by finite propagation velocity.

Accordingly, suitable basic notions are introduced. Let us consider the scalar equation in two dimension:

$$
\begin{aligned}
\partial_{t} \rho+\partial_{x} f(\rho)+\partial_{y} g(\rho) & =0, \quad t>0, \quad(x, y) \in \mathbb{R}^{2}, \\
\rho(0, \cdot) & =\rho_{0}
\end{aligned}
$$

for given functions $f, g$, and $\rho$. For systems in two dimensions, let $Q \in \mathbb{R}^{n}$, then the scalar equations (5.1) and (5.2) become a system of PDEs for two dimension given by

$$
\begin{aligned}
\partial_{t} Q+\partial_{x} F(Q)+\partial_{y} G(Q) & =0 & & \text { in } \mathbb{R}^{+} \times \mathbb{R}^{2}, \\
Q(\cdot, 0) & =Q_{0} & & \text { in } \mathbb{R}^{n} .
\end{aligned}
$$

Moreover, let us introduce the following definition.

Definition 5.1. The system is called (strictly) hyperbolic system if all eigenvalues of $\delta_{1} F^{\prime}(Q)+\delta_{2} G^{\prime}(Q)$ are real (and distinct) for all $\delta_{1}, \delta_{2} \in \mathbb{R}, Q \in \mathbb{R}^{n}$.

These eigenvalues also known as characteristic speeds, not only enable us to determine the hyperbolicity of the systems (5.1)-(5.2), but also to obtain information on the movement of the crowd.

\subsection{Qualitative properties of the model of Class I}

The class of models under consideration consists in a nonlinear, time-varying hyperbolic PDE system. Therefore, no analytical solution for this kind of systems is available, and it is known that for hyperbolic PDEs one has discontinuous solutions, namely, more than one solution at some point in time and space (for more details on hyperbolic PDEs see Ref. 26). Therefore, the model has to be written in a form that can be solved numerically to provide results that are consistent with the observed behavior.

Bearing all above in mind, the conservation form of the model (4.9) is derived, and using this form, it is proven that the hyperbolic property of the system and its isotropic nature by finding the system roots. 
Specifically, the model (4.4) is written as follows:

$$
\left\{\begin{array}{l}
\partial_{t} \rho+\partial_{x}\left(\rho v_{x}\right)+\partial_{y}\left(\rho v_{y}\right)=0 \\
\partial_{t} v_{x}+v_{x} \partial_{x} v_{x}+v_{y} \partial_{y} v_{x}=\alpha\left(v_{e}(\rho) \nu_{x_{0}}-v_{x}\right)-\frac{K^{2}(\rho)}{\rho} \partial_{x} \rho \nu_{x_{0}} \\
\partial_{t} v_{y}+v_{y} \partial_{x} v_{y}+v_{y} \partial_{y} v_{y}=\alpha\left(v_{e}(\rho) \nu_{y_{0}}-v_{y}\right)-\frac{K^{2}(\rho)}{\rho} \partial_{y} \rho \nu_{y_{0}}
\end{array}\right.
$$

which can also be rewritten as

$$
\left\{\begin{array}{l}
\partial_{t} \rho+\partial_{x}\left(\rho v_{x}\right)+\partial_{x}\left(\rho v_{x}\right)=0 \\
\rho \partial_{t} v_{x}+\rho\left[v_{x} \partial_{x} v_{x}+v_{y} \partial_{y} v_{x}\right]+K^{2}(\rho) \partial_{x} \rho \nu_{x_{0}}=\rho \mathscr{A}_{1}[\rho, \vec{v}] \\
\rho \partial_{t} v_{y}+\rho\left[v_{y} \partial_{x} v_{y}+v_{y} \partial_{y} v_{y}\right]+K^{2}(\rho) \partial_{y} \rho \nu_{y_{0}}=\rho \mathscr{A}_{2}[\rho, \vec{v}]
\end{array}\right.
$$

Some technical manipulations of (5.6) lead to the following conservation form:

$$
\begin{aligned}
& \partial_{t}\left(\rho v_{x}\right)+\partial_{x}\left(\rho v_{x}^{2}+\rho K^{2}\right)+\partial_{y}\left(\rho v_{x} v_{y}\right)=\rho \mathscr{A}_{1}[\rho, \vec{v}], \\
& \partial_{t}\left(\rho v_{y}\right)+\partial_{x}\left(\rho v_{x} v_{y}\right)+\partial_{y}\left(\rho v_{y}^{2}+\rho K^{2}\right)=\rho \mathscr{A}_{2}[\rho, \vec{v}] .
\end{aligned}
$$

Let us now look for the solution to the Riemann problem and let us write the system in the conservation form

$$
\partial_{t} Q+\partial_{x} F(Q)+\partial_{y} G(Q)=S
$$

where $Q$ is the conservative variables, $F$ and $G$ are the fluxes in the two space dimension, while $S$ is considered as the source term. These are given by

$$
\begin{gathered}
Q=\left[\begin{array}{c}
\rho \\
\rho v_{x} \\
\rho v_{y}
\end{array}\right], \quad F(Q)=\left[\begin{array}{c}
\rho v_{x} \\
\rho v_{x}^{2}+K^{2} \rho \\
\rho v_{x} v_{y}
\end{array}\right], \\
G(Q)=\left[\begin{array}{c}
\rho v_{y} \\
\rho v_{x} v_{y} \\
\rho v_{y}^{2}+K^{2} \rho
\end{array}\right], \quad S=\left[\begin{array}{c}
0 \\
\rho \mathscr{A}_{1} \\
\rho \mathscr{A}_{2}
\end{array}\right] .
\end{gathered}
$$

Next, the system is written in the quasi-linear form, while, by setting the relaxation terms to zero, the homogeneous hyperbolic partial differential equation in vector form is as follows:

$$
\partial_{t} Q+A(Q) \partial_{x} Q+B(Q) \partial_{y} Q=0
$$

where the flux Jacobian matrices $A(Q)$ and $B(Q)$ can be found from the partial derivative of the fluxes given by

$$
A:=\frac{\partial F}{\partial Q}=\left[\begin{array}{ccc}
0 & 1 & 0 \\
K^{2}-v_{v}^{2} & 2 v_{x} & 0 \\
-v_{x} v_{y} & v_{y} & v_{x}
\end{array}\right], \quad B:=\frac{\partial G}{\partial Q}=\left[\begin{array}{ccc}
0 & 0 & 1 \\
-v_{x} v_{y} & v_{y} & v_{x} \\
K^{2}-v_{y}^{2} & 0 & 2 v_{y}
\end{array}\right] .
$$


This quasi-linear matrix form of the governing equations was used to explore the characteristic structure of the system. The eigenvalues of the matrices $A$ and $B$ are found from the flux Jacobian matrices roots by solving the characteristic equation

$$
|A(Q)-\lambda I|=0
$$

that gives the eigenvalues

$$
\lambda_{1 A}=v_{x}-K, \quad \lambda_{2 A}=v_{x}, \quad \lambda_{3 A}=v_{x}+K,
$$

and the corresponding eigenvectors

$$
\vartheta_{1 A}=\left[\begin{array}{c}
1 \\
v_{x}-K \\
v_{y}
\end{array}\right], \quad \vartheta_{2 A}=\left[\begin{array}{l}
0 \\
0 \\
1
\end{array}\right], \quad \vartheta_{3 A}=\left[\begin{array}{c}
1 \\
v_{x}+K \\
v_{y}
\end{array}\right] .
$$

Referring now to the matrix $B$, the solution of the eigenvalues equation

$$
|B(Q)-\lambda I|=0,
$$

yields

$$
\lambda_{1 B}=v_{y}-K, \quad \lambda_{2 B}=v_{y}, \quad \lambda_{3 B}=v_{y}+K,
$$

while the corresponding eigenvectors

$$
\vartheta_{1 B}=\left[\begin{array}{c}
1 \\
v_{x} \\
v_{y}-K
\end{array}\right], \quad \vartheta_{2 B}=\left[\begin{array}{l}
0 \\
1 \\
0
\end{array}\right], \quad \vartheta_{3 B}=\left[\begin{array}{c}
1 \\
v_{x} \\
v_{y}+K
\end{array}\right] .
$$

Considering that the problem is in two dimensions, the overall system eigenvalues are obtained from the roots of the combined Jacobian matrices satisfying Definition 5.1. By using this information, it can be deduced that the crowd dynamic model (5.5) eigenvalues are given by

$$
\tilde{\lambda}_{1}=\tilde{v}-K\left(\delta_{1}-\delta_{2}\right), \quad \tilde{\lambda}_{2}=\tilde{v}, \quad \tilde{\lambda}_{3}=\tilde{v}-K\left(\delta_{1}+\delta_{2}\right),
$$

where $\tilde{v}=\delta_{1} v_{x}+\delta_{2} v_{y}$. The corresponding eigenvectors are

$$
\vartheta_{1 \tilde{A}}=\left[\begin{array}{c}
1 \\
v_{x}-\delta_{1} K \\
v_{y}-\delta_{2} K
\end{array}\right], \quad \vartheta_{2 \tilde{A}}=\left[\begin{array}{c}
0 \\
-\delta_{2} \\
\delta_{1}
\end{array}\right], \quad \vartheta_{3 \tilde{A}}=\left[\begin{array}{c}
1 \\
v_{x}+\delta_{1} K \\
v_{y}+\delta_{2} K
\end{array}\right] .
$$

The eigenvalues are real and distinct, and their corresponding eigenvectors are linearly independent, hence the model (4.4) is strictly hyperbolic. Finally, it can be observed that $\lambda_{1}<\lambda_{3}$, therefore the system preserves its isotropic nature.

\subsection{Qualitative properties of the Model (4.16)}

Let us now consider the system in the conservation form to find its eigenvalues. Moreover, let us focus on the derivation of the $x$-component only, since the 
$y$-component can be obtained by the same procedure. By multiplying the second equation of (4.16) by $\rho$ yields

$$
\begin{gathered}
\rho \partial_{t}\left(v_{x}+P_{1}\left(\rho, v_{x}\right) \nu_{x_{0}}\right)+\rho v_{x} \partial_{x}\left(v_{x}+P_{1}\left(\rho, v_{x}\right) \nu_{x_{0}}\right) \\
+\rho v_{y} \partial_{y}\left(v_{x}+P_{1}\left(\rho, v_{x}\right) \nu_{x_{0}}\right)=0 .
\end{gathered}
$$

Moreover, we drop $\left(\rho, v_{x}\right)$ from $P_{1}\left(\rho, v_{x}\right)$ for convenience for the rest of this subsection. It is known, from the product rule of partial differentiation, that

$$
\begin{gathered}
\partial_{t}\left(\rho\left(v_{x}+P_{1} \nu_{x_{0}}\right)\right)=\left(v_{x}+P_{1} \nu_{x_{0}}\right) \partial_{t} \rho+\rho \partial_{t}\left(v_{x}+P_{1} \nu_{x_{0}}\right), \\
\partial_{x}\left(\rho v_{x}\left(v_{x}+P_{1} \nu_{x_{0}}\right)\right)=\left(v_{x}+P_{1} \nu_{x_{0}}\right) \partial_{x}\left(\rho v_{x}\right)+\left(\rho v_{x}\right) \partial_{x}\left(v_{x}+P_{1} \nu_{x_{0}}\right), \\
\partial_{y}\left(\rho v_{y}\left(v_{x}+P_{1} \nu_{y_{0}}\right)\right)=\left(v_{x}+P_{1} \nu_{y_{0}}\right) \partial_{y}\left(\rho v_{y}\right)+\left(\rho v_{y}\right) \partial_{y}\left(v_{y}+P_{1} \nu_{y_{0}}\right) .
\end{gathered}
$$

Therefore, substituting the above terms in (5.13) and using the continuity equation to write the model in conservation form (follow same steps for the $y$-component using $P_{2}$ ), yields

$$
\begin{gathered}
\partial_{t} \rho+\partial_{x}\left(\rho v_{x}\right)+\partial_{y}\left(\rho v_{y}\right)=0 \\
\partial_{t}\left(\rho\left(v_{x}+P_{1} \nu_{x_{0}}\right)\right)+\partial_{x}\left(\rho v_{x}\left(v_{x}+P_{1} \nu_{x_{0}}\right)\right)+\partial_{y}\left(\rho v_{y}\left(v_{x}+P_{1} \nu_{x_{0}}\right)\right)=\rho \mathscr{A}_{1}[\rho, \vec{v}] \\
\partial_{t}\left(\rho\left(v_{y}+P_{2} \nu_{y_{0}}\right)\right)+\partial_{x}\left(\rho v_{x}\left(v_{y}+P_{2} \nu_{y_{0}}\right)\right)+\partial_{y}\left(\rho v_{y}\left(v_{y}+P_{2} \nu_{y_{0}}\right)\right)=\rho \mathscr{A}_{2}[\rho, \vec{v}] .
\end{gathered}
$$

These equations are also supplemented by initial conditions $\rho(0, x, y) \geq 0$ and $v_{x}(0, x) \leq\left|v_{1 x}\right|$ and $v_{y}(0, y) \leq\left|v_{2 y}\right|$. Next is to write the system in two-dimensional vector form (5.9) where $Q$ is the conservative variables, and $F$ and $G$ are the fluxes in the $x$ and $y$-directions, respectively.

The system can be written in vector form as follows:

$\partial_{t}\left[\begin{array}{c}\rho \\ \rho\left(v_{x}+P_{1} \nu_{x_{0}}\right) \\ \rho\left(v_{y}+P_{2} \nu_{y_{0}}\right)\end{array}\right]+\partial_{x}\left[\begin{array}{c}\rho v_{x} \\ \rho v_{x}\left(v_{x}+P_{1} \nu_{x_{0}}\right) \\ \rho v_{x}\left(v_{y}+P_{2} \nu_{y_{0}}\right)\end{array}\right]+\partial_{y}\left[\begin{array}{c}\rho v_{y} \\ \rho v_{y}\left(v_{x}+P_{1} \nu_{y_{0}}\right) \\ \rho v_{y}\left(v_{y}+P_{2} \nu_{y_{0}}\right)\end{array}\right]=\left[\begin{array}{c}0 \\ \rho \mathscr{A}_{1} \\ \rho \mathscr{A}_{2}\end{array}\right]$.

The eigenvalue problem can be solved by finding the flux Jacobian matrices $A$ and $B$ from the quasi-linear form (5.11). In order to get $A$ and $B$, let us state

$$
Q=(\rho, \zeta, \varrho)^{T}, \quad \zeta=\rho\left(v_{x}+P_{1} \nu_{x_{0}}\right), \quad \varrho=\rho\left(v_{y}+P_{2} \nu_{y_{0}}\right) .
$$

Substituting the new states in the fluxes yields

$$
F(Q)=\left[\begin{array}{c}
\zeta-\rho P_{1} \nu_{x_{0}} \\
\frac{\zeta^{2}}{\rho}-\zeta P_{1} \nu_{x_{0}} \\
\frac{\zeta \varrho}{\rho}-\varrho P_{1} \nu_{x_{0}}
\end{array}\right], \quad G(Q)=\left[\begin{array}{c}
\varrho-\rho P_{2} \nu_{y_{0}} \\
\frac{\zeta \varrho}{\rho}-\zeta P_{2} \nu_{y_{0}} \\
\frac{\varrho^{2}}{\rho}-\varrho P_{2} \nu_{y_{0}}
\end{array}\right]
$$


while the Jacobian matrices can be written as follows:

$$
\begin{gathered}
A(Q)=\left[\begin{array}{ccc}
-P_{1} \nu_{x_{0}}-\rho P_{1 \rho} \nu_{x_{0}} & 1-\rho P_{1 \zeta} \nu_{x_{0}} & 0 \\
-\frac{\zeta^{2}}{\rho^{2}}-\zeta P_{1 \rho} \nu_{x_{0}} & 2 \frac{\zeta}{\rho}-P_{1} \nu_{x_{0}}-\varrho P_{1 \zeta} \nu_{x_{0}} & 0 \\
-\frac{\zeta \varrho}{\rho^{2}}-\varrho P_{1 \rho} \nu_{x_{0}} & \frac{\varrho}{\rho}-\varrho P_{1 \zeta} \nu_{x_{0}} & \underline{\rho}-P_{1} \nu_{x_{0}}
\end{array}\right], \\
B(Q)=\left[\begin{array}{ccc}
-P_{2} \nu_{y_{0}}-\rho P_{2 \rho} \nu_{y_{0}} & 0 & 1-\rho P_{2 \varrho} \nu_{x_{0}} \\
-\frac{\zeta \varrho}{\rho^{2}}-\zeta P_{2 \rho} \nu_{y_{0}} & \frac{\varrho}{\rho}-P_{2} \nu_{y_{0}} & \frac{\zeta}{\rho}-\zeta P_{2 \varrho} \nu_{y_{0}} \\
-\frac{\varrho^{2}}{\rho^{2}}-\varrho P_{2 \rho} \nu_{y_{0}} & 0 & 2 \frac{\varrho}{\rho}-P_{2} \nu_{y_{0}}-\varrho P_{2 \varrho} \nu_{y_{0}}
\end{array}\right] .
\end{gathered}
$$

Therefore, a lengthy and careful calculation of the real distinct eigenvalues of $A$ gives

$$
\lambda_{1 A}=v_{x}-(\gamma+1) P_{1} \nu_{x_{0}} ; \quad \lambda_{2 A}=\lambda_{3 A}=v_{x} .
$$

Consequently, the corresponding right eigenvectors are

$$
\vartheta_{1 A}=\left[\begin{array}{c}
\frac{\beta-\rho^{\gamma+1}}{\beta v_{y}} \\
\frac{v_{x}}{v_{y}} \\
1
\end{array}\right], \quad \vartheta_{2 A}=\left[\begin{array}{c}
\frac{\left(\beta-\rho^{\gamma+1}\right)^{2}}{\beta v_{x}\left(\beta+\gamma \rho^{\gamma+1}\right)} \\
1 \\
0
\end{array}\right], \quad \vartheta_{3 A}=\left[\begin{array}{l}
0 \\
0 \\
1
\end{array}\right] .
$$

Referring to the $B(Q)$ matrix, analogous technical calculations give the following eigenvalues:

$$
\lambda_{1 B}=v_{y}-(\gamma+1) P_{2} \nu_{y_{0}} ; \quad \lambda_{2 B}=\lambda_{3 B}=v_{y},
$$

while the eigenvectors are given by

$$
\vartheta_{1 B}=\left[\begin{array}{c}
\frac{\beta-\rho^{\gamma+1}}{\beta v_{x}} \\
1 \\
\frac{v_{y}}{v_{x}}
\end{array}\right], \quad \vartheta_{2 B}=\left[\begin{array}{l}
0 \\
1 \\
0
\end{array}\right], \quad \vartheta_{3 B}=\left[\begin{array}{c}
\frac{\left(\beta-\rho^{\gamma+1}\right)^{2}}{\beta v_{y}\left(\beta+\gamma \rho^{\gamma+1}\right)} \\
0 \\
1
\end{array}\right] .
$$

The eigenvalues can be computed by solving the combined Jacobian matrices satisfying definition (5.1). Specifically, the eigenvalues are found to be

$$
\tilde{\lambda}_{1}=\tilde{v}_{x}-(\gamma+1) P_{1} \nu_{x_{0}}, \quad \tilde{\lambda}_{2}=\tilde{\lambda}_{3}=\tilde{v}_{x}
$$

while their corresponding eigenvectors are given by

$$
\vartheta_{1 \tilde{A}}=\left[\begin{array}{c}
\frac{\beta-\rho^{\gamma+1}}{\beta v_{y}} \\
\frac{v_{x}}{v_{y}} \\
1
\end{array}\right], \quad \vartheta_{2 \tilde{A}}=\left[\begin{array}{c}
\delta_{1} \frac{\left(\beta-\rho^{\gamma+1}\right)^{2}}{\beta \tilde{v}_{x}\left(\beta+\gamma \rho^{\gamma+1}\right)} \\
1 \\
0
\end{array}\right], \quad \vartheta_{3 \tilde{A}}=\left[\begin{array}{c}
\delta_{2} \frac{\left(\beta-\rho^{\gamma+1}\right)^{2}}{\beta \tilde{v}_{x}\left(\beta+\gamma \rho^{\gamma+1}\right)} \\
0 \\
1
\end{array}\right] .
$$

These eigenvalues are real and two of them repeated. Nevertheless, we are able to find linearly independent eigenvectors, and therefore the crowd dynamic model obtained is a nonlinear hyperbolic system of partial differential equation. In addition, the pressure term is an increasing function of density, and from the eigenvalue 
$\tilde{\lambda}_{1}$, we are sure to have the maximum wave speed to be $\tilde{v}$. Thus, the model has the desired anisotropic property.

\subsection{Qualitative properties of the model of Class III: (4.17)-(4.19)}

The conservation form is derived by expanding the derivatives in the first equation of (4.1) as follows:

$$
\rho\left(\partial_{x} v_{x}+\partial_{y} v_{y}\right)=-\left(\partial_{t} \rho+v_{x} \partial_{x} \rho+v_{y} \partial_{y} \rho\right),
$$

where for $v_{e}(\rho) \nu_{x_{0}}$, one has:

$$
\begin{gathered}
v_{e}^{\prime}(\rho) \nu_{x_{0}} \partial_{t} \rho=\partial_{t} v_{e}(\rho) \nu_{x_{0}}, \quad v_{e}^{\prime}(\rho) \nu_{x_{0}} \partial_{x} \rho=\partial_{x} v_{e}(\rho) \nu_{x_{0}}, \\
v_{e}^{\prime}(\rho) \nu_{y_{0}} \partial_{y} \rho=\partial_{y} v_{e}(\rho) \nu_{y_{0}} .
\end{gathered}
$$

Exploiting the above calculations for system (4.18), after having multiplied by $\rho$, yields:

$$
\rho \partial_{t}\left(v_{x}-v_{e}(\rho) \nu_{x_{0}}\right)+\rho v_{x} \partial_{x}\left(v_{x}-v_{e}^{\prime}(\rho) \nu_{x_{0}}\right)+\rho v_{y} \partial_{y}\left(v_{x}-v_{e}^{\prime}(\rho) \nu_{x_{0}}\right)=\rho \mathscr{A}_{1} .
$$

Moreover, using the product rules yields:

$$
\begin{gathered}
\partial_{t}\left(\rho\left(v_{x}-v_{e}(\rho) \nu_{x_{0}}\right)\right)=\partial_{t} \rho\left(v_{x}-v_{e}(\rho) \nu_{x_{0}}\right)+\rho \partial_{t}\left(v_{x}-v_{e}(\rho) \nu_{x_{0}}\right), \\
\partial_{x}\left(\rho v_{x}\left(v_{x}-v_{e}(\rho) \nu_{x_{0}}\right)\right)=\partial_{x}\left(\rho v_{x}\right)\left(v_{x}-v_{e}(\rho) \nu_{x_{0}}\right)+\left(\rho v_{x}\right) \partial_{x}\left(v_{x}-v_{e}(\rho) \nu_{x_{0}}\right), \\
\partial_{y}\left(\rho v_{y}\left(v_{x}-v_{e}(\rho) \nu_{x_{0}}\right)\right)=\partial_{x}\left(\rho v_{y}\right)\left(v_{x}-v_{e}(\rho) \nu_{x_{0}}\right)+\left(\rho v_{y}\right) \partial_{y}\left(v_{x}-v_{e}(\rho) \nu_{x_{0}}\right) .
\end{gathered}
$$

Next, substituting in (5.19), and using the conservation law (4.11) leads, after some manipulations, to the equation in conservative form as follows:

$$
\begin{gathered}
\partial_{t}\left(\rho\left(v_{x}-v_{e}(\rho) \nu_{x_{0}}\right)\right)+\partial_{x}\left(\rho v_{x}\left(v_{x}-v_{e}(\rho) \nu_{x_{0}}\right)\right) \\
+\partial_{y}\left(\rho v_{y}\left(v_{x}-v_{e}(\rho) \nu_{x_{0}}\right)\right)=\rho \mathscr{A}_{1}, \\
\partial_{t}\left(\rho\left(v_{y}-v_{e}(\rho) \nu_{y_{0}}\right)\right)+\partial_{x}\left(\rho v_{x}\left(v_{y}-v_{e}(\rho) \nu_{y_{0}}\right)\right) \\
\quad+\partial_{y}\left(\rho v_{y}\left(v_{y}-v_{e}(\rho) \nu_{y_{0}}\right)\right)=\rho \mathscr{A}_{2},
\end{gathered}
$$

supplemented by initial conditions are $\rho(0, x, y) \geq 0$ and $v_{x}(0, x) \leq\left|v_{1 x}\right|$ and $v_{y}(0, y) \leq\left|v_{2 y}\right|$.

Next, the system is written in the two-dimensional vector form (5.9), where $Q$ is the conservative variables, and $F$ and $G$ are the fluxes in the $x$ - and $y$-directions respectively, and $S$ can be considered as the source term. The PDE system as follows:

$$
\partial_{t}\left[\begin{array}{c}
\rho \\
\rho\left(v_{x}-v_{e}(\rho) \nu_{x_{0}}\right) \\
\rho\left(v_{y}-v_{e}(\rho) \nu_{y_{0}}\right)
\end{array}\right]+\partial_{x}\left[\begin{array}{c}
\rho v_{x} \\
\rho v_{x}\left(v_{x}-v_{e}(\rho) \nu_{x_{0}}\right) \\
\rho v_{x}\left(v_{y}-v_{e}(\rho) \nu_{y_{0}}\right)
\end{array}\right]+\partial_{y}\left[\begin{array}{c}
\rho v_{y} \\
\rho v_{y}\left(v_{x}-v_{e}(\rho) \nu_{x_{0}}\right) \\
\rho v_{y}\left(v_{y}-v_{e}(\rho) \nu_{y_{0}}\right)
\end{array}\right]=S,
$$

where $S \equiv\left[\begin{array}{lll}0 & \rho \mathscr{A}_{1} & \rho \mathscr{A}_{2}\end{array}\right]^{\mathrm{T}}$ denote the source vector. 
The eigenvalue problem can be solved by applying the same method as in the previous subsection. Therefore, we rewrite the system into the conservative form and compute the fluxes Jacobian matrices $A$ and $B$. In order to do so, let us state:

$$
\zeta=\rho\left(v_{x}+v_{e}(\rho) \nu_{x_{0}}\right), \quad \varrho=\rho\left(v_{y}+v_{e}(\rho) \nu_{y_{0}}\right) .
$$

Next, substituting the new states in the fluxes yields

$$
F(Q)=\left[\begin{array}{c}
\zeta+\rho v_{e}(\rho) \nu_{x_{0}} \\
\frac{\zeta^{2}}{\rho}+\zeta \rho v_{e}(\rho) \nu_{x_{0}} \\
\frac{\zeta \varrho}{\rho}+\varrho \rho v_{e}(\rho) \nu_{x_{0}}
\end{array}\right], \quad G(Q)=\left[\begin{array}{c}
\varrho+\rho v_{e}(\rho) \nu_{y_{0}} \\
\frac{\zeta \varrho}{\rho}+\zeta v_{e}(\rho) \nu_{y_{0}} \\
\frac{\varrho^{2}}{\rho}+\varrho v_{e}(\rho) \nu_{y_{0}}
\end{array}\right]
$$

Therefore, the Jacobian matrices are as follows:

$$
\begin{gathered}
A(Q)=\left[\begin{array}{ccc}
v_{e}(\rho) \nu_{x_{0}}+\rho v_{e}(\rho) \nu_{x_{0}} & 1 & 0 \\
-\frac{\zeta^{2}}{\rho^{2}}+\zeta v_{e}(\rho) \nu_{x_{0}} & 2 \frac{\zeta}{\rho}+v_{e}(\rho) \nu_{x_{0}} & 0 \\
-\frac{\zeta \varrho}{\rho^{2}}+\varrho v_{e}^{\prime}(\rho) \nu_{x_{0}} & \underline{\varrho} & \frac{\zeta}{\rho}+v_{e}(\rho) \nu_{x_{0}}
\end{array}\right], \\
B(Q)=\left[\begin{array}{ccc}
v_{e}(\rho) \nu_{y_{0}}+\rho v_{e}^{\prime}(\rho) \nu_{y_{0}} & 0 & 1 \\
-\frac{\zeta \varrho}{\rho^{2}}+\zeta v_{e}^{\prime}(\rho) \nu_{y_{0}} & \underline{\varrho}+v_{e}(\rho) \nu_{y_{0}} & \frac{\zeta}{\rho} \\
-\frac{\varrho^{2}}{\rho^{2}}+\zeta v_{e}^{\prime}(\rho) \nu_{y_{0}}(\rho) & 0 & 2 \frac{\varrho}{\rho}+v_{e}(\rho) \nu_{y_{0}}
\end{array}\right] .
\end{gathered}
$$

Computing the eigenvalues for the matrix $A$ yields

$$
\lambda_{1 A}=v_{x}+\rho v_{e}^{\prime}(\rho) \nu_{y_{0}}, \quad \lambda_{2 A}=\lambda_{3 A}=v_{x},
$$

while the eigenvectors are given by

$$
\vartheta_{1 A}=\left[\begin{array}{c}
1 \\
v_{x}-\vec{v}_{e} \\
v_{y}-v_{e}(\rho) \nu_{y_{0}}
\end{array}\right], \quad \vartheta_{2 A}=\left[\begin{array}{c}
1 \\
v_{x}-\vec{v}_{e}-\rho v_{e}^{\prime}(\rho) \nu_{x_{0}} \\
0
\end{array}\right], \quad \vartheta_{3 A}=\left[\begin{array}{l}
0 \\
0 \\
1
\end{array}\right] .
$$

Similarly for the matrix $B$, one has for the eigenvalues:

$$
\lambda_{1 B}=v_{y}-\rho v_{e}^{\prime}(\rho) \nu_{y_{0}}, \quad \lambda_{2 B}=\lambda_{3 B}=v_{y},
$$

and for eigenvectors:

$$
\vartheta_{1 B}=\left[\begin{array}{c}
1 \\
v_{x}-v_{e}(\rho) \nu_{x_{0}} \\
v_{y}-v_{e}(\rho) \nu_{y_{0}}
\end{array}\right], \quad \vartheta_{2 B}=\left[\begin{array}{l}
0 \\
1 \\
0
\end{array}\right], \quad \vartheta_{3 B}=\left[\begin{array}{c}
1 \\
0 \\
v_{y}-v_{e}(\rho) \nu_{y_{0}}-\rho v_{e}^{\prime}(\rho) \nu_{y_{0}}
\end{array}\right] .
$$

Since the system involves two dimension, one needs to verify that it is hyperbolic, that the eigenvalues found earlier are valid for any combination of the roots of the 
combined system as defined by Definition 5.1. The eigenvalues are computed as follows:

$$
\lambda_{1}=\tilde{v}_{x}+\rho \tilde{v}_{e}^{\prime}, \quad \lambda_{2}=\lambda_{3}=\tilde{v}_{x},
$$

where $\tilde{v}=\delta_{1} v_{x}+\delta_{2} v_{y}$ and $\tilde{v}_{e}^{\prime}=\delta_{1} v_{x}(\rho) \nu_{x_{0}}+\delta_{2} v_{y}(\rho) \nu_{y_{0}}$. Considering that these eigenvalues are real, it can be concluded that the model is hyperbolic, and that, considering that we also have repeated eigenvalues, the model is not strictly hyperbolic. For each eigenvalue the corresponding eigenvectors are given by

$$
\vartheta_{1 \tilde{A}}=\left[\begin{array}{c}
1 \\
v_{x}-v_{e}(\rho) \nu_{x_{0}} \\
v_{y}-v_{e}(\rho) \nu_{y_{0}}
\end{array}\right], \quad \vartheta_{2 \tilde{A}}=\left[\begin{array}{c}
1 \\
\frac{\tilde{v}-v_{e}(\rho) \nu_{x_{0}}-\rho \tilde{v}_{e}(\rho) \nu_{x_{0}}}{\delta_{1}} \\
0
\end{array}\right], \quad \vartheta_{3 \tilde{A}}=\left[\begin{array}{c}
0 \\
-\frac{\delta_{1}}{\delta_{2}} \\
1
\end{array}\right] .
$$

\subsection{Comparison between the models}

The system (4.4), or equivalently the system (5.5), is strictly hyperbolic, because the calculation of the eigenvalues based on the gradient computation of the flux matrices gives three distinguished values. This result holds except for $K=0$, where two eigenvalues coalesce, and the matrices $A$ and $B$ cannot any longer be diagonalized.

Therefore, the solutions of (4.4) can be found, in this model no internal inconsistency appears. Models of Class I are characterized by an isotropic nature since one of the eigenvalues is always moving faster than the velocity itself. Consequently, the partial differential system (4.9), implemented with proper initial/boundary data, generates a well-posed mathematical problem.

The second class of models uses two-coupled PDEs with an anticipation term $K(\rho)$ and the relaxation terms $\mathscr{A}$. These terms change the momentum equation to mimic crowd dynamic flow. The anticipation factors role is to find the macroscopic response of pedestrian to traffic density, namely, its response when interaction between pedestrians and obstacles are overlooked within their domain. By the solution of an eigenvalue problem, one deduces that this model has an anisotropic nature since one of its eigenvalues is always moving faster than the velocity itself.

The behavior of the systems (4.17)-(4.19) is qualitatively similar to those models of Class I. In this class of models, we replace the relaxation term with a convective derivative on the pressure term. The class of model has three real and two repeated eigenvalues. The system is hyperbolic and shows an anisotropic nature that is evident from its eigenvalues, where $\lambda_{1} \leq \lambda_{2}=\lambda_{3}$ for both velocity directions. This means that all information moves at a speed equal or less than the velocity of the corresponding state.

All above results are summarized in Table 1.

\section{Additional Analysis}

The contents of this paper, after a preliminary analysis of scaling and representation problems, has been focused on modelling crowd dynamics at the macroscopic scale. This final section aims at developing a critical analysis toward further developments of the modelling approach. In details, the following topics, selected among several 
Table 1. On the consistency of the class of models.

\begin{tabular}{|c|c|c|c|}
\hline \multirow[b]{2}{*}{ Characteristic } & \multicolumn{3}{|c|}{ Models } \\
\hline & Class I & Class III.A & Class III.B \\
\hline Conservative variables $Q$ & $\rho, \rho v_{x}, \rho v_{y}$ & $\begin{array}{c}\rho, \rho\left(v_{x}+P_{1} \nu_{x_{0}}\right) \\
\rho\left(v_{y}+P_{2} \nu_{y_{0}}\right)\end{array}$ & $\begin{array}{c}\rho, \rho\left(v_{x}-\rho v_{e}^{\prime} \nu_{x_{0}}\right) \\
\rho\left(v_{y}-\rho v_{e}^{\prime} \nu_{x_{0}}\right)\end{array}$ \\
\hline "Pressure" term $P$ & $\rho K(\rho)=\rho K_{0}^{2}$ & $\frac{\vec{v} \rho^{\gamma+1}}{\beta-\rho^{\gamma+1}}, \begin{array}{l}\gamma>0 \\
\beta>\rho m^{\gamma+1}\end{array}$ & $-\rho v_{e}^{\prime}(\rho) \nu_{x_{0}}=\frac{\rho v_{x}}{\rho_{m}}$ \\
\hline \multicolumn{4}{|c|}{$X$-direction $F(Q)$ eigenvalues } \\
\hline$\lambda_{1}$ & $v_{x}-K_{0}$ & $v_{x}-(\gamma+1) P_{1} \nu_{x_{0}}$ & $v_{x}+\rho v_{e}^{\prime} \nu x_{0}$ \\
\hline$\lambda_{2}$ & $v_{x}$ & $v_{x}$ & $v_{x}$ \\
\hline$\lambda_{3}$ & $v_{x}+K_{0}$ & $v_{x}$ & $v_{x}$ \\
\hline \multicolumn{4}{|c|}{$Y$-direction $G(Q)$ eigenvalues } \\
\hline$\lambda_{1}$ & $v_{y}-K_{0}$ & $v_{y}-(\gamma+1) P_{2} \nu_{y_{0}}$ & $v_{y}+\rho v_{e}^{\prime} \nu y_{0}$ \\
\hline$\lambda_{2}$ & $v_{y}$ & $v_{y}$ & $v_{y}$ \\
\hline$\lambda_{3}$ & $v_{y}+K_{0}$ & $v_{y}$ & $v_{y}$ \\
\hline PDE type & Strictly Hyperbolic & Hyperbolic & Hyperbolic \\
\hline Isotropic & Yes & No & No \\
\hline Antisotropic & No & Yes & Yes \\
\hline
\end{tabular}

conceivable ones, are treated in the subsections below: statement of mathematical problems, transition from normal to panic situations, modelling swarms, and finally a critical analysis related to development of multiscale methods. Each of the above issues are dealt with at an introductory level, while a deeper analysis needs to be properly developed within a suitable research plan.

\subsection{Statement of mathematical problems}

Let us consider, the statement of mathematical problems for the class of models reported in the preceding sections. Typically, problems can be classified into initial value problems in unbounded domains and initial-boundary value problems in bounded domains.

The initial value problem for models of crowd dynamics (3.1) is stated in unbounded domains for active particles (individuals) who have the objective to reach a point of the whole space. The statement is obtained by linking the models to initial conditions for the density and velocity variables:

$$
\rho_{0}(\mathbf{x}, \mathbf{v})=\rho(t=0, \mathbf{x}, \mathbf{v}), \quad \vec{q}_{0}(\mathbf{x}, \mathbf{v})=\vec{q}(t=0, \mathbf{x}, \mathbf{v}), \quad \forall \mathbf{x} \in D_{t_{0}} \subset \mathbb{R}^{2}, \quad \forall \mathbf{v} \in D_{\mathbf{v}},
$$

where $D_{t_{0}}$ is the domain of the space variable where the initial condition is localized, see Fig. 3.

The solution to the above problem should provide the evolution $D_{t}$ of the domain occupied by the individuals starting from the domain $D_{t_{0}}$ occupied at the initial time. If individuals have a target to pursue, the problem is meaningful if the target $T$ does not belong to the domain of the initial conditions and until $T$ is not included in $D_{t_{0}}$. 


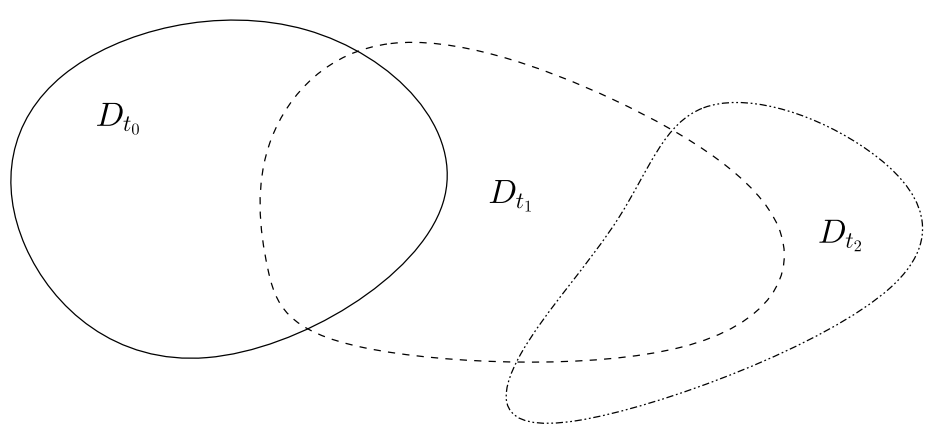

Fig. 3. Time evolution of the domain occupied by the crowd.

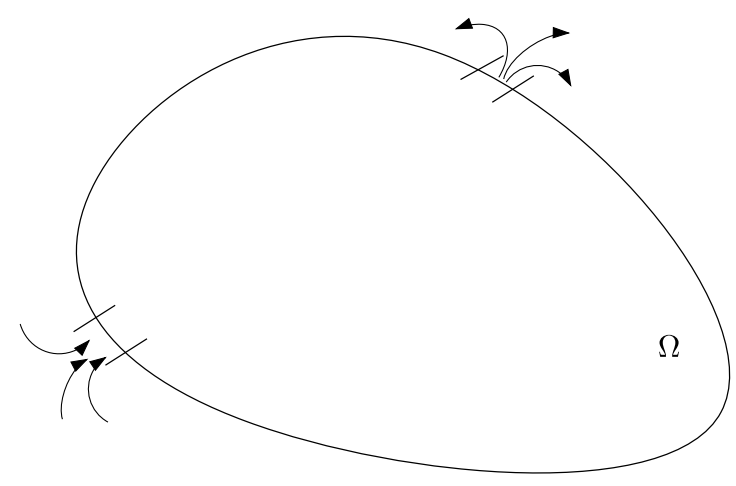

Fig. 4. Closed domain with inlet and outlet boundaries.

Let us now consider the statement of boundary conditions in a fixed domain $\Omega$ with boundary $\partial \Omega$. Moreover, let $\Omega_{T}$ be the part of the boundary where particles flow out, and $\Omega_{I}$ be the part where the inlet of individuals occur. Finally, let us denote by $\partial \Omega_{b}$ the part of the boundary where no inlet or outlet occurs, see Fig. 4 , and assume that $\partial \Omega_{b}$ is regular so that the normal to the surface $\vec{n}$ can be defined. The statement of the boundary conditions has to be considered for each of the boundary domains $\mathscr{T}, \mathscr{I}$ and $\Omega_{b}$.

The statement on $\partial \Omega_{b}$ is obtained by assuming that the flow is equal to zero:

$$
\vec{q} \cdot \vec{n}=0, \quad \forall \mathbf{x} \in \partial \Omega_{p} .
$$

For other parts of the boundary, it can be assumed that the flow is prescribed:

$$
\begin{cases}\vec{q} \cdot \vec{n}=v_{I}, & \forall \mathbf{x} \in \partial \Omega_{I}, \\ \vec{q} \cdot \vec{n}=v_{T}, & \forall \mathbf{x} \in \partial \Omega_{T},\end{cases}
$$

or that not gradients are allowed along the inlet or outlet zone:

$$
\nabla_{\vec{n}}(\vec{q} \cdot \vec{n})=0, \quad \forall \mathbf{x} \in \partial \Omega_{I}, \quad \text { or } \forall \mathbf{x} \in \partial \Omega_{T}
$$


The above statement is reasonable in the case of normal flow, while it should be properly adapted to the case of panic situations. Moreover, one has to take into account unavoidable fluctuations. Therefore, it is very important to analyze the sensitivity of the solution to the mathematical problem with respect to perturbation in the data and the production terms.

\subsection{Transition from regular to panic conditions}

The behavior of crowds in panic situations appears to be substantially different from that observed in normal flow conditions. In some cases panic behaviors generate extremely dangerous situations which may possibly be avoided by suitable control of the behavior of the pedestrians: their reaction to panic signals, and to the behavior of surrounding individuals.

The analysis will be focused on models derived within the frameworks proposed in the preceding sections referring both to the collective strategy and to individual interactions. As already mentioned, the topic is not treated extensively, but some qualitative indications, focused on modelling panic conditions, are given with research perspectives:

1. Individuals are not subject to the acceleration directed toward a precise objective, while they follow the presence of the other particles.

2. Interactions among individuals substantially differ from those in normal flow conditions as attracting accelerations become predominant with respect to repulsive. Moreover, their intensity becomes relatively greater.

3. The individual behavior tends to be the same for all individuals as all of them are aware of the environmental situation, thus reducing behavioral differences from individual to individual.

Some of the models proposed in Sec. 4 can be properly modified to take into account the aforementioned behaviors. The first modification refers to modelling the equilibrium velocity, as both expressions (4.5) and (4.6) need to be modified. Specifically, $V_{M}$ substantially increased in panic conditions, while suitable experiments (not yet available) should be developed to model $v_{e}$ in panic conditions.

The second modification refers to the attraction toward density gradients, that changes sign in panic conditions. Namely, the crowd is attracted by positive density gradients rather than negative. Therefore, a correction using $\vec{\mu}_{M}$ rather than $\vec{\mu}_{m}$ should be used. Moreover, this effect becomes quantitatively relevant with respect to the relaxation toward the equilibrium velocity.

The above considerations already provide some preliminary suggestions toward a modelling approach although experiments, not yet available, appear to be necessary.

\subsection{Modelling swarms}

Modelling of swarms is an attractive research perspective which may be also motivated by the observation of the beauty of the shapes formed by birds which appear 
in the sky during spring and autumn periods. The modelling process needs to be related to the statement of mathematical problems which is an initial value problem, while the domain containing the swarm evolves in time.

Generally, a swarm does not have a precise target to reach unless it has to escape a danger signal appearing from the outer environment. However, one may expect that paths of minimum density gradients are followed, while interactions at the microscopic scale follow rules somehow similar to those of crowds. Moreover, the behavior on the border of the swarm follows rules different from those of particles well inside the swarm.

Bearing all above in mind, some guide lines will be given toward a modelling project:

1. Mathematical problems are stated in unbounded domains with initial conditions with compact support. The solution to those problems should provide the evolution in time of the domain of the initial conditions.

2. Individuals are not subject to the acceleration directed toward a precise objective, while modelling of microscopic interactions should also take into account the localization of the interacting pairs. The output of the interactions is different from the border to the center of the swarm.

3. The swarm generally has one or more leading individuals so that the others are attracted by leaders who may stochastically modify their trajectories.

Transferring the above ideas to a modelling approach is definitely a challenging problem that may even require adopting a representation at a different scale, specifically the one offered by the kinetic theory. ${ }^{2}$ A hybrid approach, definitely worth developing consists in adding to the macroscopic description a perturbation induced by long-range interactions. In all cases with respect to crowd dynamics a different self-organization ability has to be considered.

\subsection{Scaling problems}

The modelling approach proposed in this paper is based on the macroscopic description of mass and linear momentum equations properly closed by phenomenological models of the acceleration acting on the crowd elementary volume in each point of the space domain.

It is obvious that the system under consideration does not satisfy the classical continuum assumption. Therefore, macroscopic models have to be considered as an approximation of physical reality that in some cases, for instance low density regimes, may not be satisfactory. Moreover, this is not the only drawback of the macroscopic approach, as models are derived under the assumption that all individuals behave in the same way, namely the system is not heterogeneous. Therefore, the approach of this paper should be regarded as a first step to be followed by additional analysis focused on different modelling methods. 
On the other hand, it is not naively claimed that the use of the other scales is fully satisfactory. Various criticisms can be raised for each of the scales defined in Sec. 2. For instance, models at the microscopic scale involve not only difficulties in modelling individual behaviors, but also computational complexity induced by the large number of differential equations and by the subsequent difficulty in developing the averaging process. The system under consideration is characterized by complex dynamics, where microscopic interactions play the role, while macroscopic information is needed to describe the overall behavior.

Methods of the generalized kinetic theory for active particles can be used. This approach, documented in the book, ${ }^{2}$ includes an additional variable - called activity - in the microscopic variable that models the individual behavior of the interacting elements, called active particles. This approach has the advantage of describing the statistical distribution of individual behavior, that may depend on the local flow conditions. On the other hand, even in high density conditions the number of individuals in the crowd is never large enough to justify the continuous approximation of the distribution function. Two recent papers devoted to vehicular traffic flow modelling, ${ }^{8,11}$ suggest to discretize the velocity space so that the assumption of continuity over the velocity variable is technically relaxed. Of course, the same reasoning should be applied to the space and activity variables.

On the other hand, macroscopic models should be considered as models which are characterized by a lower computational complexity with respect to models derived according to suitable developments of the kinetic theory. Indeed, these models allow quite immediate application as documented in the paper by Coscia and Canavesio. ${ }^{7}$ However, previous studies in other fields of living sciences, e.g. cellular dynamics in biology, can be properly developed to derive macroscopic equations from the underlying microscopic description, delivered by kinetic type models, as documented in papers 3 and 4.

The above reasonings about scaling problem, the suggestion to develop kinetic type models in a discrete space of microscopic states, and to derive macroscopic models from the kinetic theory description, can be extended also to modelling swarms. Indeed, it appears to be an interesting research perspective.

\section{References}

1. A. Aw and M. Rascle, Resurrection of "second order" models of traffic flow, SIAM J. Appl. Math. 60 (2000) 916-938.

2. N. Bellomo, Modelling Complex Living Systems - A Kinetic Theory and Stochastic Game Approach (Birkhäuser, 2008).

3. N. Bellomo and A. Bellouquid, On the onset of nonlinearity for diffusion models of binary mixtures of biological materials by asymptotic analysis, Int. J. Non-Linear Mech. 41 (2006) 281-293.

4. N. Bellomo, A. Bellouquid, J. Nieto and J. J. Soler, Multicellular growing systems: Hyperbolic limits toward macroscopic description, Math. Mod. Meth. Appl. Sci. 17 (2007) 1675-1693. 
5. N. Bellomo, A. Marasco and A. Romano, From the modelling of driver's behavior to hydrodynamic models and problems of traffic flow, Nonlinear Anal. RWA 3 (2002) 339-363.

6. E. Bonabeau, M. Dorigo and G. Theraulaz, Swarm Intelligence: From Natural to Artificial Systems (Oxford Univ. Press, 1999).

7. V. Coscia and C. Canavesio, First order macroscopic modelling of human crowd dynamics, Math. Mod. Meth. Appl. Sci. 18 (2008) 1217-1247.

8. V. Coscia, M. Delitala and P. Frasca, On the mathematical theory of vehicular traffic flow models II. Discrete velocity kinetic models, Int. J. Nonlinear Mech. 42 (2007) 411-421.

9. P. Degond and M. Delitala, Modelling and simulation of vehicular jam formation, Kinetic Rel. Mod. 1 (2008) 279-293.

10. J. M. Del Castillo and G. G. Benitez, On the funtional form of the speed-density relationship-I: Transport. Res. Part B 29 B (1993) 373-389.

11. M. Delitala and A. Tosin, Mathematical modelling of vehicular traffic: A discrete kinetic theory approach, Math. Mod. Meth. Appl. Sci. 17 (2007) 901-932.

12. C. Dogbé, On the numerical solutions of second order macroscopic models of pedestrian flows, Comp. Math. with Appl. to appear.

13. L. F. Henderson, The statistics of crowd fluids, Nature 229 (1971) 381-383.

14. L. F. Henderson, On the fluid mechanic of human crowd motions, Transp. Res. 8 (1975) 509-515.

15. D. Helbing, A mathematical model for the behavior of pedestrians, Behavioral Sci. 36 (1991) 298-310.

16. D. Helbing, A fluid-dynamic model for the movement of pedestrians, Complex Syst. 6 (2002) 391-415.

17. D. Helbing and P. Molnár, Social force model for pedestrians dynamics, Phys. Rev. E 51 (1995) 4282-4286.

18. D. Helbing and P. Molnár, Walker model for the formation of human and animal trail systems, Phys. Rev. E 56 (1997) 2527-2539.

19. D. Helbing, I. Farkas and T. Vicsek, Simulating dynamical feature of excape panic, Nature 407 (2000) 487-490.

20. D. Helbing, Traffic and related self-driven many-particle systems, Rev. Mod. Phys. 73 (2001) 1067-1141.

21. S. Hoogendoorn and P. H. L. Bovy, Simulation of pedestrian flows by optimal control and differential games, Opt. Cont. Appl. Meth. 23 (2003) 153-172.

22. R. L. Hughes, A continuum theory for the flow of pedestrians, Transport. Res. Part B 36 (2002) 507-536.

23. R. L. Hughes, The flow of human crowds, Ann. Rev. Fluid Mech. 35 (2003) 169-183.

24. A. Klar, R. D. Küne, R. Wegener, Mathematical models for vehicular traffic, Surv. Math. Ind. 6 (1996) 215-239.

25. J. P. Lebacque and M. M. Khoshyaran, First order traffic flow models: Intersection modelling, network modelling, application, Int. Symp. Transport Traffic Flow Theory (2005).

26. R. J. Leveque, Finite Volume Methods for Hyperbolic Problems (Cambridge Univ. Press, 2002).

27. M. J. Lighthill and G. B. Whitham, On the kinematic waves II. A theory of traffic flow on long crowded roads, Proc. Roy. Soc. London A 199 (1975) 317-345.

28. H. J. Payne, Models of freeway traffic and control, in Mathematical Models of Public Systems 1, G. A. Bekey, ed., Simulation Council Proceedings Series, La Jolla, California (1971), pp. 51-61. 
29. P. I. Richards, Shock waves on the highway, Operat. Res. 4 (1956) 42-51.

30. F. Venuti, L. Bruno and N. Bellomo, Crowd dynamics on a moving platform: Mathematical modelling and application to lively footbridges, Math. Comp. Model. 45 (2007) 252-269.

31. M. Zhang, A non-equilibrium traffic flow model devoid of gas-like behavior, Transp. Res. Part B 36 (2002) 275-290.

32. M. Zhang, A theory of nonequilibrium traffic flow, Transp. Res. Part B 32 (1998) 485-498. 